\title{
A Novel Regiospecific Synthesis of 1-Chloro-2-arylcyclohexenes
}

\author{
Lokesh Krishnappa and HariPrasad Suresh \\ Department of Studies in Chemistry, Central College Campus, Bangalore University, Bangalore, Karnataka 560 001, India
}

Correspondence should be addressed to HariPrasad Suresh; hariprasad@bub.ernet.in

Received 23 February 2014; Accepted 2 April 2014; Published 9 July 2014

Academic Editor: William Setzer

Copyright (C) 2014 L. Krishnappa and H. Suresh. This is an open access article distributed under the Creative Commons Attribution License, which permits unrestricted use, distribution, and reproduction in any medium, provided the original work is properly cited.

An efficient high yielding chemoselective synthesis of eleven novel 1-chloro-2-arylcyclohexenes employing the Suzuki cross coupling of 1-bromo-2-chlorocyclohexene with eleven different aryl boronic acids and $\mathrm{Pd}(\mathrm{dppf}) \mathrm{Cl}_{2} \cdot \mathrm{CH}_{2} \mathrm{Cl}_{2}$ catalyst is reported.

\section{Introduction}

Cross-coupling reactions of unsaturated carbon centres bearing halogens/triflates with aryl boronic acids catalyzed by palladium metal complexes have become a powerful tool in organic synthesis for the formation of carbon-carbon bonds. One of the important cross-coupling reactions in this genre is the Suzuki reaction [1-4]. These organopalladium complex catalyzed couplings of unsaturated halides with boronic acids/esters have been employed in key steps to form a wide variety of pharmaceuticals and natural products [510].

1,2-Dihalocycloalkenes are precursors to cycloalkynes $[11,12]$. However, there are very few reports for the conversion of 1,2-dihalocycloalkenes to substituted 1-halo-2arylcycloalkenes. One report shows the chemoselective cross coupling of 2-bromo-3-chloronorbornadiene to 2-chloro-3aryl-norbornadiene [13]. In this regard, the Suzuki coupling reaction of cyclohexenyl bromides with arylboronic acids is still almost unexplored. A recent report shows its importance in the synthesis of polysubstituted phenol derivatives [14].

Our laboratory is involved in the synthesis and reactions of some novel cyclic vinyl silanes. We employ the WurtzFittig coupling reaction of corresponding cyclic vinyl halide with sodium and chlorotrimethylsilane in suitable solvent to prepare the cyclic vinylsilanes. Using the Wurtz-Fittig reaction, we have been able to synthesize a large number of simple and substituted cyclic vinylsilanes [15-19]. The organosilicon compounds are anionic synthons serving as the starting materials in the total synthesis of natural products [20-23].
Cyclic vinyl halides and 1,2-dihalocycloalkenes serve as precursors to the cyclic vinylsilanes. There are several protocols for the preparation of cyclic vinyl halides and 1,2-dihalocycloalkenes [24]. The protocols for 1,2dihalocycloalkenes normally afford the two halogen atoms symmetrically attached to the carbon-carbon double bond $[25,26]$. In comparison to the preparation of symmetrical 1,2-dihalocycloalkenes, there exist very few protocols for the synthesis of unsymmetrical 1,2-dihalocycloakenes especially 1-bromo-2-chlorocycloalkenes [27-31]. We had earlier adopted the addition reaction of bromine chloride to 1chlorocyclopentene followed by dehydrochlorination for the synthesis of 1-bromo-2-chlorocyclopentene [25].

In this paper, we report for the first instance the synthesis of 1-bromo-2-chlorocyclohexene (2) and the chemoselective Suzuki cross-coupling reaction with eleven different aryl boronic acids (3a-k) to form eleven novel 1-chloro-2arylcyclohexenes $(\mathbf{4 a - k})$ in high yields.

\section{Materials and Methods}

All reactions were performed in oven dried apparatus. Reactions were monitored on Merck F-254 precoated TLC plastic sheets using hexane $\left(60-80^{\circ} \mathrm{C}\right)$ fraction as mobile phase. GC was run on SE-30 SS $2 \mathrm{~m} \times 1 / 8^{\prime \prime}$ column on Mayura 9800 gas chromatograph. IR spectra were recorded on Shimadzu FTIR $8400 \mathrm{~S}$ using $\mathrm{NaCl}$ flats as neat thin liquid film samples. The values are reported in wave numbers $\left(\mathrm{cm}^{-1}\right) .{ }^{1} \mathrm{H}$ NMR and ${ }^{13} \mathrm{C}$ NMR were obtained on a Bruker AMX 400 spectrometer using $\mathrm{CDCl}_{3}$ with tetramethylsilane as internal standard. 
Chemical shifts are reported in $\delta$ (ppm downfield from tetramethylsilane). Coupling constants are reported in $\mathrm{Hz}$ with multiplicities denoted as $\mathrm{s}$ (singlet), $\mathrm{d}$ (doublet), $\mathrm{t}$ (triplet), q (quartet), m (multiplet), and br (broad). GC-MS spectra were obtained using a Shimadzu GC-MS QP 5050A instrument equipped with a $30 \mathrm{~mm} \times 0.32 \mathrm{~mm}$ BP-5 capillary column. Elemental analyses were obtained using Elementar Vario Microcube-15106062 instrument. All yields refer to the isolated yields of the products.

2.1. Synthesis of 1-Bromo-2-chlorocyclohexene (2). To a stirred suspension of $\mathrm{PCl}_{5}(1.4 \mathrm{~g}, 6.723 \mathrm{mmol})$ in $10 \mathrm{~mL}$ anhydrous ether was added a $10 \mathrm{~mL}$ ethereal solution of 1 (1.0 g, $5.525 \mathrm{mmol}$ ), dropwise over $30 \mathrm{~min}$. The mixture was stirred for further 8 hours, when all the starting material disappeared as followed by GC. The reaction mixture was poured into crushed ice $(50 \mathrm{~g})$ and extracted with ether $(3 \times 30 \mathrm{~mL})$. The combined organic extracts were washed with brine $(20 \mathrm{~mL})$, dried over anhydrous $\mathrm{Na}_{2} \mathrm{SO}_{4}$, and concentrated in vacuo to isolate $1.05 \mathrm{~g}$ of concentrate.

To $0.63 \mathrm{~g}(5.614 \mathrm{mmol})$ of potassium tert-butoxide in $10 \mathrm{~mL}$ tert-butyl alcohol was added the $1.05 \mathrm{~g}$ of crude in $10 \mathrm{~mL}$ tert-butyl alcohol drop wise over $15 \mathrm{~min}$ and refluxed for 2 hours, when the aliquot indicated a single peak in gas chromatogram. The mixture was diluted with water $(150 \mathrm{~mL})$, extracted with ether $(4 \times 30 \mathrm{~mL})$, washed with brine $(20 \mathrm{~mL})$, dried (an. $\mathrm{Na}_{2} \mathrm{SO}_{4}$ ), and concentrated. Distillation under reduced pressure gave pure 2 (0.62 g, 56\%).

2.2. General Procedure for the Synthesis of 1-Chloro2-arylcyclohexenes $(\mathbf{4 a}-\boldsymbol{k})$. A mixture of 1-bromo-2chlorocyclohexene 2 (196 mg, $1.0 \mathrm{mmol})$, aryl boronic acid $(1.0 \mathrm{mmol}), \mathrm{K}_{2} \mathrm{CO}_{3}(3.0 \mathrm{mmol})$, and $\mathrm{Pd}(\mathrm{dppf}) \mathrm{Cl}_{2} \cdot \mathrm{CH}_{2} \mathrm{Cl}_{2}$ $(5 \mathrm{~mol} \%)$ in $10 \mathrm{~mL} \mathrm{1,4-dioxane} \mathrm{was} \mathrm{heated} \mathrm{in} \mathrm{a} \mathrm{sealed} \mathrm{tube}$ placed in an oil bath at $70-80^{\circ} \mathrm{C}$ for $2-3 \mathrm{hr}$. The reaction mixture was cooled to ambient temperature and filtered, and the solvent was removed in vacuo. The crude product was then subjected to column chromatography using silica gel(60-100 mesh) and 1:10 ethyl acetate/hexane $\left(60-80^{\circ} \mathrm{C}\right.$ fraction) to isolate $\mathbf{4 a - k}$ in greater than $90 \%$ yield (Table 2 ).

\subsection{Spectral Data for the Products}

2.3.1. 1-Bromo-2-chlorocyclohexene (2). IR: 2941, 2862, 2839, $1641,1434,1325,1006 \mathrm{~cm}^{-1} ;{ }^{1} \mathrm{H} \operatorname{NMR}\left(\mathrm{CDCl}_{3}\right) \delta: 1.70-1.80(\mathrm{~m}$, $4 \mathrm{H}), 2.40-2.45(\mathrm{~m}, 2 \mathrm{H}) 2.53-2.58(\mathrm{~m}, 2 \mathrm{H}) ;{ }^{13} \mathrm{C} \mathrm{NMR}\left(\mathrm{CDCl}_{3}\right)$ $\delta: 23.30,23.89,34.60,36.47,119.50,130.83$; GC-MS m/e (rel. int.): 198 (0.29), 196 (1.22), 194 (0.80), 168 (0.18), 166 (0.17), 160 (0.42), 159 (0.33), 158 (0.27), 129 (0.24), 117 (2.63), 116 (1.06), 115 (7.30), 114 (0.72), 113 (0.57), 100 (0.94), 99 (1.14), 89 (1.16), 87 (3.19), 81 (2.18), 80 (8.05), 79 (100.00), 78 (7.21), 77 (29.9), 75 (3.88), 74 (3.40), 73 (3.66), 63 (7.03), 53 (5.90), 52 (12.56), 51 (34.76), 50 (20.39) 49 (4.31); Anal. Found: C, 36.95; H, 4.19. Calcd: C, 36.86; H, 4.12 .

2.3.2.1-Chloro-2-phenylcyclohexene (4a). IR: 698, 1004, 1440, 2858, $2929 \mathrm{~cm}^{-1} ;{ }^{1} \mathrm{H}$ NMR $\left(\mathrm{CDCl}_{3}\right) \delta: 1.70-2.84(\mathrm{~m}, 4 \mathrm{H})$, $2.36-2.47(\mathrm{~m}, 2 \mathrm{H}), 2.48-2.57(\mathrm{~m}, 2 \mathrm{H}), 7.24-7.60(\mathrm{~m}, 5 \mathrm{H}) ;{ }^{13} \mathrm{C}$ $\mathrm{NMR}\left(\mathrm{CDCl}_{3}\right) \delta: 22.68,23.90,32.95,34.09,126.09,127.15$,
127.23, 127.80, 128.03, 128.73, 134.40, 141.56; Anal. Found: C, 74.76; H, 6.75. Calcd: C, 74.80; H, 6.80.

2.3.3. 1-Chloro-2-phenyl-3'-methyl-cyclohexene (4b). IR: 1440, 1693, 2880, $2931 \mathrm{~cm}^{-1} ;{ }^{1} \mathrm{H}$ NMR $\left(\mathrm{CDCl}_{3}\right) \delta: 1.69-1.84$ $(\mathrm{m}, 4 \mathrm{H}), 2.35(\mathrm{~s}, 3 \mathrm{H}), 2.36-2.58(\mathrm{~m}, 4 \mathrm{H}), 7.04-7.59(\mathrm{~m}$, $4 \mathrm{H}) ;{ }^{13} \mathrm{C} \mathrm{NMR}\left(\mathrm{CDCl}_{3}\right) \delta: 22.70,23.34,23.93,33.02,34.64$, 119.56, 124.26, 125.12, 127.67, 127.94, 128.67, 137.59, 141.54; Anal. Found: C, 75.45; H, 7.27. Calcd: C, 75.53; H, 7.31.

2.3.4.1-Chloro-2-phenyl-4' -formyl-cyclohexene (4c). IR: 1209, 1602, 1701, 2731, 2837, 2860, $2933 \mathrm{~cm}^{-1} ;{ }^{1} \mathrm{H} \mathrm{NMR}\left(\mathrm{CDCl}_{3}\right) \delta$ : $1.26-1.87(\mathrm{~m}, 4 \mathrm{H}), 2.38-2.41(\mathrm{~m}, 2 \mathrm{H}), 2.48-2.52(\mathrm{~m}, 2 \mathrm{H}), 7.41-$ $7.44(\mathrm{~d}, 2 \mathrm{H}, J=12 \mathrm{~Hz}) 7.85-7.88(\mathrm{~d}, 2 \mathrm{H}, J=12 \mathrm{~Hz}), 10.00(\mathrm{~s}, 1 \mathrm{H})$; ${ }^{13} \mathrm{C} \mathrm{NMR}\left(\mathrm{CDCl}_{3}\right) \delta: 22.48,23.74,32.52,34.06,128.36,129.23$, 129.60, 130.05, 130.26, 133.52, 135.02, 147.93; GC-MS m/e (rel. int.): 221.93 (32), 219.82 (100), 184.99 (28), 154.88 (32). Anal. Found: C, 70.77; H, 5.99. Calcd: C, 70.75; H, 5.94 .

2.3.5. 1-Chloro-2-phenyl-2'-methoxy-cyclohexene (4d). IR: 1249, 1490, 1596, 2837, 2856, $2931 \mathrm{~cm}^{-1} ;{ }^{1} \mathrm{H} \mathrm{NMR}\left(\mathrm{CDCl}_{3}\right) \delta$ : 1.75-1.85 (m, 4H), 2.51 (br, 4H), 3.83 (s, 3H), 6.92-6.99 (m, 2H), 7.10-7.12 (m, 1H), 7.26-7.30 (m, 1H); ${ }^{13} \mathrm{C} \mathrm{NMR}\left(\mathrm{CDCl}_{3}\right)$ $\delta: 22.50,23.97,31.65,33.71,55.62,111.15,120.41,128.28,128.36$, 130.00, 130.57, 132.60, 156.26; Anal. Found: C, 70.15; H, 6.72; Calcd: C, 70.11; H, 6.79.

2.3.6. 1-Chloro-2-phenyl-2' -methoxy-5' -isopropyl-cyclohexene (4e). ${ }^{1} \mathrm{H} \mathrm{NMR}\left(\mathrm{CDCl}_{3}\right) \delta: 1.23-1.25(\mathrm{~d}, 6 \mathrm{H}, J=6.8 \mathrm{~Hz}), 1.75-$ 1.90 (br m, 4H), 2.10-2.35 (br, 4H), 2.40-2.60 (br, 2 H), 2.822.92 (sep, $1 \mathrm{H}), 3.79$ (s, 3H), 6.83-6.85 (d, $1 \mathrm{H}, J=8.4 \mathrm{~Hz})$, 6.96-6.97 (d, $1 \mathrm{H}, J=2.4 \mathrm{~Hz}), 7.10-7.12(\mathrm{~m}, 1 \mathrm{H}) ;{ }^{13} \mathrm{C} \mathrm{NMR}$ $\left(\mathrm{CDCl}_{3}\right) \delta: 22.99,24.45,24.59,30.13,32.17,33.67,34.23,56.24$, $111.48,126.32$, 128.56, 128.71, 130.73, 133.40, 141.18, 154.80; Anal. Found: C, 72.59; H, 7.97. Calcd: C, 72.57; H, 7.99.

2.3.7. 1-Chloro-2-phenyl-4'-ethoxy-cyclohexene (4f). IR: 1286, $1685,2862,2935 \mathrm{~cm}^{-1} ;{ }^{1} \mathrm{H}$ NMR $\left(\mathrm{CDCl}_{3}\right) \delta: 1.40-1.43(\mathrm{t}, 3 \mathrm{H}$, $J=7.2 \mathrm{~Hz}), 1.74-1.82(\mathrm{~m}, 4 \mathrm{H}), 2.35-2.49(\mathrm{~m}, 4 \mathrm{H}), 4.01-4.06$ (q, $2 \mathrm{H}, J=6.8 \mathrm{~Hz}), 6.86-6.88(\mathrm{~d}, 2 \mathrm{H}, J=8.4 \mathrm{~Hz}), 7.18-7.20$ (d, $2 \mathrm{H}, J=8.8 \mathrm{~Hz}$ ); GC-MS m/e (rel. int.): 237.80 (38), 235.81 (100), 207.70 (28), 200.82 (36), 172.76 (56). Anal. Found: C, 71.09; H, 7.30. Calcd: C, 71.03; H, 7.24.

2.3.8. 1-Chloro-2-phenyl-3', $4^{\prime}$-dimethoxy-cyclohexene (4g). IR: 1159, 1207, 1504, 1606, 2837, $2933 \mathrm{~cm}^{-1} ;{ }^{1} \mathrm{H}$ NMR $\left(\mathrm{CDCl}_{3}\right)$ $\delta$ : 1.17-1.18 (broad $\mathrm{m}, 4 \mathrm{H}$ ), 2.1-2.4 (broad s, 2H), 2.4-2.6 (broad s, 2H), 3.7 (s, 3H), $3.8(\mathrm{~s}, 3 \mathrm{H}), 6.5$ (s, 2H), 6.98-7.01 $(\mathrm{d}, 1 \mathrm{H}, J=12 \mathrm{~Hz}),{ }^{13} \mathrm{C} \mathrm{NMR}\left(\mathrm{CDCl}_{3}\right) \delta: 22.59,24.01,31.89$, 33.82, 55.28, 55.63, 99.00, 104.19, 106.19, 128.48, 130.39, 132.29, 157.35, 160.01; Anal. Found: C, 66.50; H, 6.72; Calcd: C, 66.53; $\mathrm{H}, 6.78$.

2.3.9. 1-Chloro-2-phenyl-3'-acetyl-cyclohexene (4h). IR: 1286, $1685,2862,2935 \mathrm{~cm}^{-1} ;{ }^{1} \mathrm{H}$ NMR $\left(\mathrm{CDCl}_{3}\right) \delta: 1.73-1.86(\mathrm{~m}$, $4 \mathrm{H}), 2.38-2.42(\mathrm{~m}, 2 \mathrm{H}), 2.48-2.51(\mathrm{~m}, 2 \mathrm{H}), 2.61(\mathrm{~s}, 3 \mathrm{H}), 7.46$ (s, $1 \mathrm{H}), 7.42-7.48(\mathrm{~m}, 1 \mathrm{H}) 7.85-7.86(\mathrm{q}, 2 \mathrm{H}) ;{ }^{13} \mathrm{C} \mathrm{NMR}\left(\mathrm{CDCl}_{3}\right)$ $\delta: 22.57,23.81,26.64,32.77,34.04,126.91,128.11,128.34,128.77$, 
TABLE 1: Suzuki cross coupling of $\mathbf{2}$ with $\mathbf{3 a}$ using different palladium catalysts/solvents.

\begin{tabular}{|c|c|c|c|c|c|c|}
\hline \multirow[b]{2}{*}{ Entry } & \multirow[b]{2}{*}{ Catalyst } & \multirow[b]{2}{*}{ Base } & \multirow[b]{2}{*}{ Ligand } & \multirow[b]{2}{*}{ Solvent } & \multicolumn{2}{|c|}{$\%$ Yield } \\
\hline & & & & & & $\mathrm{Cl}$ \\
\hline 01 & $\mathrm{Pd}(\mathrm{OAc})_{2}$ & $\mathrm{~K}_{2} \mathrm{CO}_{3}$ & $\mathrm{PPh}_{3}$ & Ethanol & 40 & - \\
\hline 02 & $\mathrm{Pd}(\mathrm{OAc})_{2}$ & $\mathrm{~K}_{2} \mathrm{CO}_{3}$ & $\mathrm{P}(t-\mathrm{Bu})_{3}$ & Ethanol & 25 & - \\
\hline 03 & $\mathrm{Pd}(\mathrm{OAc})_{2}$ & TEA & $\mathrm{P}(o \text {-tol })_{3}$ & Ethanol & 30 & - \\
\hline 04 & $\left(\mathrm{PPh}_{3}\right)_{2} \mathrm{PdCl}_{2}$ & $\mathrm{~K}_{2} \mathrm{CO}_{3}$ & $\mathrm{PPh}_{3}$ & Toluene & 35 & 5 \\
\hline 05 & $\left(\mathrm{PPh}_{3}\right)_{2} \mathrm{PdCl}_{2}$ & $\mathrm{~K}_{2} \mathrm{CO}_{3}$ & $\mathrm{P}(o \text {-tol })_{3}$ & Ethanol & 25 & 10 \\
\hline 06 & $\left(\mathrm{PPh}_{3}\right)_{2} \mathrm{PdCl}_{2}$ & $\mathrm{Cs}_{2} \mathrm{CO}_{3}$ & $\mathrm{P}(t-\mathrm{Bu})_{3}$ & Toluene & 30 & 15 \\
\hline 07 & $\mathrm{Pd}\left(\mathrm{PPh}_{3}\right)_{2} \mathrm{Cl}_{2}$ & TEA & $\mathrm{P}(o \text {-tol })_{3}$ & Dioxane & 35 & 30 \\
\hline 08 & $\mathrm{Pd}\left(\mathrm{PPh}_{3}\right)_{4}$ & $\mathrm{Cs}_{2} \mathrm{CO}_{3}$ & - & Dioxane & 20 & 45 \\
\hline \multirow[t]{2}{*}{09} & \multirow{2}{*}{$\mathrm{Pd}(\mathrm{dppf}) \mathrm{Cl}_{2} \cdot \mathrm{CH}_{2} \mathrm{Cl}_{2}{ }^{*}$} & \multirow{2}{*}{$\mathrm{K}_{2} \mathrm{CO}_{3}$} & \multirow{2}{*}{ - } & \multirow{2}{*}{ Dioxane } & 20 & 60 \\
\hline & & & & & $<5$ & $>90$ \\
\hline
\end{tabular}

${ }^{*}$ Reactions were carried out both at reflux condition and in sealed tube.

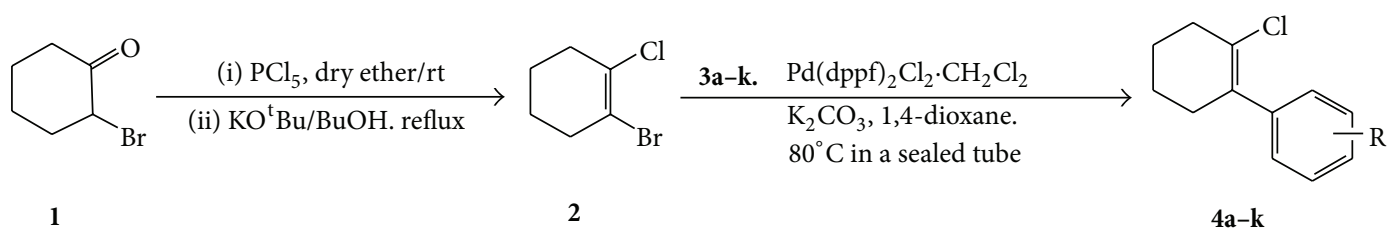

SCHEME 1

132.98, 133.56, 137.05, 141.94, 198.04; Anal. Found: C, 71.60; H, 6.40. Calcd: C, 71.64; H, 6.44 .

2.3.10. 1-Chloro-2-phenyl-3'-chloro-cyclohexene (4i). IR: $1438,1703,2860,2935 \mathrm{~cm}^{-1} ;{ }^{1} \mathrm{H}$ NMR $\left(\mathrm{CDCl}_{3}\right) \delta: 1.69-$ $1.84(\mathrm{~m}, 4 \mathrm{H}), 2.33-2.58(\mathrm{~m}, 4 \mathrm{H}), 7.12-7.58(\mathrm{~m}, 3 \mathrm{H}) ;{ }^{13} \mathrm{C}$ $\mathrm{NMR}\left(\mathrm{CDCl}_{3}\right) \delta: 23.34,23.94,34.64,36.51,119.56,126.38$, 127.04, 127.88, 128.30, 129.34, 130.12, 130.89; Anal. Found: C, 63.49; H, 5.38. Calcd. C, 63.46; H, 5.33.

2.3.11. 1,3', $4^{\prime}$-Trichloro-2-phenyl-cyclohexene (4j). ${ }^{1} \mathrm{H} \mathrm{NMR}$ $\left(\mathrm{CDCl}_{3}\right) \delta: 1.72-1.84(\mathrm{~m}, 4 \mathrm{H}), 2.32-2.35(\mathrm{~m}, 2 \mathrm{H}), 2.45-2.49$ $(\mathrm{m}, 2 \mathrm{H}), 7.08-7.11(\mathrm{q}, 1 \mathrm{H}, J=4 \mathrm{~Hz}) 7.35-7.63(\mathrm{~m}, 2 \mathrm{H}) ;{ }^{13} \mathrm{C}$ NMR $\left(\mathrm{CDCl}_{3}\right) \delta: 22.49,23.71,32.61,34.04,126.15,127.72$, 128.80, 129.33, 130.07, 130.21, 130.96, 141.34; Anal. Found: C, 55.15; H, 4.29; Calcd. C, 55.10; H, 4.24.

2.3.12. 1-Chloro-2-(2'-benzo[b]furano)-cyclohexene $(\mathbf{4 k}) .{ }^{1} \mathrm{H}$ NMR $\left(\mathrm{CDCl}_{3}\right) \delta: 1.82-1.84(\mathrm{~m}, 4 \mathrm{H}), 2.61-2.73(\mathrm{~m}, 4 \mathrm{H}), 7.23-$ $7.32(\mathrm{~m}, 3 \mathrm{H}), 7.47-7.49(\mathrm{~d}, 1 \mathrm{H}, J=7.6 \mathrm{~Hz}), 7.60-7.62(\mathrm{~d}$, $1 \mathrm{H}, J=7.6 \mathrm{~Hz}) ;{ }^{13} \mathrm{C} \mathrm{NMR}\left(\mathrm{CDCl}_{3}\right) \delta: 22.39,23.99,28.703$, $35.82,106.85,111.35,121.50,123.14,124.62,124.67,129.18,131.34$, 153.97, 154.38; Anal. Found: C, 72.30; H, 5.68. Calcd. C, 72.26; H, 5.63 .

\section{Results and Discussion}

Reaction of 2-bromocyclohexanone (1) [32-35] with $\mathrm{PCl}_{5}$ in anhydrous ether gave a mixture of chloro-compounds [26], which without further purification was subjected to dehydrohalogenation using potassium tert-butoxide/tert-butyl alcohol to isolate pure $\mathbf{2}$ in $50 \%$ overall yield from $\mathbf{1}$.

The nonsymmetrical 1-bromo-2-chlorocyclohexene $\mathbf{2}$ was subjected to the Suzuki cross-coupling reaction with phenyl boronic acid (3a) in the presence of palladium acetate catalyst [36]. The reaction however yielded biphenyl as the major product, instead of the expected cross-coupled product 1-chloro-2-phenylcyclohexene (4a).

Literature survey indicated several reports which show the use of differing palladium catalysts for the cross-coupling reactions. Some of the catalysts reported are $\mathrm{Pd}(\mathrm{OAc})_{2}$ [36], $\mathrm{PdCl}_{2}$ [37], $\left(\mathrm{PPh}_{3}\right)_{2} \mathrm{PdCl}_{2}$ [38], $\left(\mathrm{PPh}_{3}\right)_{4} \mathrm{Pd}$ [39], $\mathrm{Pd}(\mathrm{dppf}) \mathrm{Cl}_{2}$ [40], and $\mathrm{Pd}(\mathrm{dppf}) \mathrm{Cl}_{2} \cdot \mathrm{CH}_{2} \mathrm{Cl}_{2}$ [41]. We employed all the above catalysts for the Suzuki cross-coupling reaction in an effort to prepare 1-chloro-2-arylcyclohexenes $\mathbf{4 a - k}$ (Table 1).

Our investigations have shown that the best catalyst for the cross-coupling reaction of 2 to be $\mathrm{Pd}(\mathrm{dppf}) \mathrm{Cl}_{2} \cdot \mathrm{CH}_{2} \mathrm{Cl}_{2}$ under inert nitrogen atmosphere and sealed tube conditions (Scheme 1).

In a typical reaction $2(1 \mathrm{mmol})$, phenylboronic acid $3 \mathbf{a}(1 \mathrm{mmol})$, potassium carbonate $(3 \mathrm{mmol})$, and $\mathrm{Pd}(\mathrm{dppf}) \mathrm{Cl}_{2} \cdot \mathrm{CH}_{2} \mathrm{Cl}_{2} \quad(5 \mathrm{~mol} \%)$ in $8-10 \mathrm{~mL}$ 1,4-dioxane solvent were heated on an oil bath at $80^{\circ} \mathrm{C}$ in a sealed tube for 3 hours. Monitoring using TLC and GC indicated the completion of reaction. The reaction mixture was filtered and subjected to column chromatography using silica gel (60-100 mesh) and 1:10 ethyl acetate/hexane $\left(60-80^{\circ} \mathrm{C}\right.$ fraction) to isolate $\mathbf{4 a}$ in $95 \%$ isolated yield.

The reaction of $\mathbf{2}$ was extended to a wide variety of aryl boronic acids $\mathbf{3 b}-\mathbf{k}$ to isolate the products $\mathbf{4 b}-\mathbf{k}$. Each 
TABle 2: Cross coupling of 1-bromo-2-chlorocyclohexene (2) with differing aryl boronic acids (3a-k). Preparation of novel 1-chloro-2arylcyclohexenes (4a-k).

\begin{tabular}{|c|c|c|c|c|}
\hline Entry & $\begin{array}{c}\text { Aryl boronic acids } \\
(\mathbf{3 a}-\mathbf{k})\end{array}$ & $\begin{array}{l}\text { Products } \\
(\mathbf{4 a}-\mathbf{k})\end{array}$ & Time (hr) & $\%$ Yield \\
\hline 01 & $\mathrm{OH})_{2}$ & $4 a$ & 3 & 95 \\
\hline 02 & $\mathrm{OH})_{2}$ & b & 2.5 & 90 \\
\hline 03 & $\mathrm{~B}(\mathrm{OH})$ & & 3 & 91 \\
\hline 04 & $3(\mathrm{OH})_{2}$ & & 2 & 93 \\
\hline 05 & $\mathrm{OH})$ & $4 e$ & 2 & 90 \\
\hline 06 & $3 f$ & & 2 & 92 \\
\hline 07 & $(\mathrm{OH})_{2}$ & & 2 & 95 \\
\hline 08 & $\mathrm{~B}(\mathrm{OH})_{2}$ & 41 & 3 & 95 \\
\hline 09 & $\mathrm{~B}(\mathrm{OH})_{2}$ & $4 \mathrm{i}$ & 2.5 & 94 \\
\hline 10 & $\mathrm{~B}(\mathrm{OH})_{2}$ & $4 j$ & 2.5 & 92 \\
\hline
\end{tabular}


TABLE 2: Continued.

Entry $\begin{gathered}\text { Aryl boronic acids } \\ (\mathbf{3 a - k})\end{gathered}$

coupling reaction was repeated for a minimum of three trials and the optimum yields of the products are indicated in Table 2 . All products $\mathbf{4 a - k}$ were characterised completely by IR, ${ }^{1} \mathrm{H}-\mathrm{NMR},{ }^{13} \mathrm{C}-\mathrm{NMR}, \mathrm{GC}-\mathrm{MS}$, and elemental analysis.

The mechanism for the formation of the 1-chloro-2arylcyclohexenes may be explained based on the bond dissociation energies of the carbon-bromine and carbonchlorine which are 289 and $335 \mathrm{~kJ} \mathrm{moL}^{-1}$, respectively. In the first step, due to the lower bond energy between carbon and bromine, oxidative addition of the $\mathrm{Pd}(\mathrm{dppf}) \mathrm{Cl}_{2} \cdot \mathrm{CH}_{2} \mathrm{Cl}_{2}$ catalyst to 2 occurs across the carbon-bromine bond. In presence of potassium carbonate/arylboronic acid $\mathbf{3 a - k}$, the species eliminates $\mathrm{KBr}, \mathrm{CO}_{2}$, and $\mathrm{KOB}(\mathrm{OH})_{2}$ followed by reductive elimination of the 1-chloro-2-arylcyclohexene $4 \mathbf{a}-$ k. The restored palladium catalyst further continues the cycle.

\section{Conclusion}

A general methodology for the regiospecific replacement of bromine in 1-bromo-2-chlorocycloalkenes and formation of some novel 1-chloro-2-arylcyclohexenes using the Suzuki cross-coupling reaction are reported. The method is simple and cost efficient and yields the 1-chloro-2- aryl-cyclohexenes in high yields in short duration of time.

\section{Conflict of Interests}

The authors declare that there is no conflict of interests regarding the publication of this paper.

\section{Acknowledgments}

The authors thank the University Grants Commission Grant no. F. 36-42/2008 (SR) and Government of India, New Delhi, for financial assistance. Grateful thanks are placed on record to the Indian Institute of Science, Bangalore, for providing NMR facility.

\section{References}

[1] K. C. Nicolaou, P. G. Bulger, and D. Sarlah, "Palladiumcatalyzed cross-couplingreactions in total synthesis," Angewandte Chemie International Edition, vol. 44, no. 29, pp. 44424489, 2005.

[2] D. S. Jason, T. A. Derek, J. A. G. Thomas, and G. D. Abigail, "Enantioselective, nickel-catalyzed Suzuki cross-coupling of Quinolinium ions," Organic Letters, vol. 16, no. 1, pp. 142-145, 2014.
[3] P.-L. Moisés, "Palladium nanoparticles as efficient catalysts for Suzuki cross-coupling reactions," The Journal of Physical Chemistry Letters, vol. 3, no. 2, pp. 167-174, 2012.

[4] J. D. St. Denis, C. C. G. Scully, C. F. Lee, and A. K. Yudin, "Development of the direct Suzuki-Miyaura cross-coupling of primary b-alkyl MIDA-boronates and aryl bromides," Organic Letters, vol. 16, no. 5, pp. 1338-1341, 2014.

[5] R. Jana, T. P. Pathak, and M. S. Sigman, "Advances in transition metal $(\mathrm{Pd}, \mathrm{Ni}, \mathrm{Fe})$-catalyzed cross-coupling reactions using alkyl-organometallics as reaction partners," Chemical Reviews, vol. 111, no. 3, pp. 1417-1492, 2011.

[6] Á. Molnár, "Efficient, selective, and recyclable palladium catalysts in carbon-carbon coupling reactions," Chemical Reviews, vol. 111, no. 3, pp. 2251-2320, 2011.

[7] C. Liu, H. Zhang, W. Shi, and A. Lei, "Bond formations between two nucleophiles: transition metal catalyzed oxidative crosscoupling reactions," Chemical Reviews, vol. 111, no. 3, pp. 17801824, 2011.

[8] S. Theeramunkong, A. Caldarelli, A. Massarotti et al., "Regioselective Suzuki coupling of dihaloheteroaromatic compounds as a rapid strategy to synthesize potent rigid combretastatin analogues," Journal of Medicinal Chemistry, vol. 54, no. 14, pp. 4977-4986, 2011.

[9] C.-S. Chan, A. K.-S. Tse, and K. S. Chan, "Highly versatile methods for the synthesis of quinonylporphyrins via benzannulation of Fischer carbene complexes and palladium-catalyzed crosscoupling reactions," The Journal of Organic Chemistry, vol. 59, no. 20, pp. 6084-6089, 1994.

[10] F. Guillier, F. Nivoliers, A. Godard et al., "Combined metalationpalladium-catalyzed cross coupling strategies. A formal synthesis of the marine alkaloid amphimedine," The Journal of Organic Chemistry, vol. 60, no. 2, pp. 292-296, 1995.

[11] N. Suzuki, M. Nishiura, and Y. Wakatsuki, "Isolation and structural characterization of 1-zirconacyclopent-3-yne, fivemembered cyclic alkynes," Science, vol. 295 , no. 5555, pp. $660-$ $663,2002$.

[12] K. Komatsu, S. Aonuma, Y. Jinbu, R. Tsuji, C. Hirosawa, and K. Takeuchi, "Generation and oligomerization of bicyclo[2.2.2] octyne and properties of tris(bicyclo[2.2.2]octeno)benzene obtained from the linear trimer," The Journal of Organic Chemistry, vol. 56, no. 1, pp. 195-203, 1991.

[13] W.-J. Yoo, G. C. Tsui, and W. Tam, "Palladium-catalyzed Suzuki couplings of 2,3-dibromonorbornadiene: synthesis of symmetrical and unsymmetrical aryl-substituted norbornadienes," European Journal of Organic Chemistry, no. 6, pp. 1044-1051, 2005.

[14] W. T. Spencer III and A. J. Frontier, "Cycloaromatization protocol for synthesis of polysubstituted phenol derivatives: method development and mechanistic studies," The Journal of Organic Chemistry, vol. 77, no. 17, pp. 7730-7736, 2012. 
[15] G. Nagendrappa, "Synthesis of 1-trimethylsilylcycloalkenes from 1-bromocycloalkenes by Wurtz-type coupling," Synthesis, vol. 1980, no. 9, pp. 704-706, 1980.

[16] S. HariPrasad and G. Nagendrappa, "Vinylsilanes in synthesis: 2-halo-1-cyclopentenyl alkyl/aryl ketones from 2-halo-1trimethylsilylcyclopentenes," Indian Journal of Chemistry B: Organic and Medicinal Chemistry, vol. 36, no. 8, pp. 691-694, 1997.

[17] S. HariPrasad and G. Nagendrappa, "Preparation of amino substituted cyclic vinylsilanes by Wurtz-Fittig procedure," Indian Journal of Chemistry B: Organic and Medicinal Chemistry, vol. 36, no. 11, pp. 1016-1019, 1997.

[18] D. Jyothi and S. HariPrasad, "Cyclic $\alpha$-acylvinyl anionic synthons: a novel synthesis of 2-trimethylsilyl-3-methyl-cyclohexenone by the Wurtz-Fittig coupling reaction," Synthetic Communications, vol. 39, no. 5, pp. 875-879, 2009.

[19] D. Jyothi and S. HariPrasad, "Five- and six-membered cyclic $\alpha$-acylvinyl anionic synthons: synthesis of $\alpha$-trimethylsilyl$\alpha, \beta$-unsaturated cycloalkenones and their conversion into 2-(hydroxydimethylsilyl)cycloalk-2-enones through carbonsilicon bond scission," Arkivoc, no. 6, pp. 194-203, 2012.

[20] E. Langkopf and D. Schinzer, "Uses of silicon-containing compounds in the synthesis of natural products," Chemical Reviews, vol. 95, no. 5, pp. 1375-1408, 1995.

[21] P. Wang, X.-L. Yeo, and T.-P. Loh, "Copper-catalyzed highly regioselective silylcupration of terminal alkynes to form $\alpha$ vinylsilanes," Journal of the American Chemical Society, vol. 133, no. 5, pp. 1254-1256, 2011.

[22] A. Vadalà, P. V. Finzi, G. Zanoni, and G. Vidari, "Columbetdione, a new cyclopentene derivative from the fruiting bodies of Tricholoma columbetta (basidiomycetes)-structure and synthesis," European Journal of Organic Chemistry, no. 4, pp. 642-648, 2003.

[23] Y. Ishihara, A. Mendoza, and P. S. Baran, "Total synthesis of taxane terpenes: cyclise phase," Tetrahedron, vol. 69, no. 27-28, pp. 5685-5701, 2013.

[24] A. Krebs and J. Wilke, "Angle strained cycloalkynes," in Wittig Chemistry, vol. 109 of Topics in Current Chemistry, pp. 189-233, 1983.

[25] S. HariPrasad and G. Nagendrappa, "Reactions of 1,2dihalocycloalkenes with alkali metals in presence of chlorotrimethylsilane. Reductive carbon-carbon bond cleavage in five membered homocyclic system," Tetrahedron, vol. 49, no. 16, pp. 3387-3396, 1993.

[26] K. Griesbaum, R. Kiber, and B. Pfeffer, "Synthese und stabilität von 2,3-dichloroxiranen," Justus Liebigs Annalen der Chemie, vol. 1975, no. 2, pp. 214-224, 1975.

[27] A. Favorskii, "Sur la triple liaison dans les cycles carbones et sur la structure possible des plus simples hydrocarbures cycliques $\mathrm{C}_{\mathrm{n}} \mathrm{H}_{\mathrm{n}-4}$," Bulletin de la Société Chimique de France, pp. 1727$1735,1936$.

[28] N. Domnin, "Sur la structure possible de l'hydrocarbure $\mathrm{C}_{7} \mathrm{H}_{10}$ a cycle heptagonal," Bulletin de la Société Chimique de France, pp. 1735-1739, 1936.

[29] G. Wittig and R. Pohlke, "Zur existenz niedergliedriger cycloalkine, II," Chemische Berichte, vol. 94, no. 12, pp. 32763286, 1961.

[30] A. T. Blomquist, R. E. Burge Jr., and A. C. Sucsy, "Manymembered carbon rings. V. Cyclodecyne, cis- and transcyclodecene and related compounds," Journal of the American Chemical Society, vol. 74, no. 14, pp. 3636-3642, 1952.
[31] J. von Braun and H. Ostermayer, "Zur Kenntnis der halogenderivate des indens," Berichte der Deutschen Chemischen Gesellschaf B, vol. 70, no. 5, pp. 1006-1008, 1937.

[32] A. R. Jagdale, P. V. Chouthaiwale, and A. Sudalai, "Cu(OTf) catalyzed $\alpha$-halogenation of ketones with 1,3-dichloro-5, $5^{\prime}$ dimethylhydantoin and $\mathrm{N}$-bromosuccinimide," Indian Journal of Chemistry B: Organic and Medicinal Chemistry, vol. 48, no. 10, pp. 1424-1430, 2009.

[33] I. Pravst, M. Zupan, and S. Stavber, "Halogenation of ketones with $\mathrm{N}$-halosuccinimides under solvent-free reaction conditions," Tetrahedron, vol. 64, no. 22, pp. 5191-5199, 2008.

[34] S. Adimurthy, S. Ghosh, P. U. Patoliya et al., "An alternative method for the regio- and stereoselective bromination of alkenes, alkynes, toluene derivatives and ketones using a bromide/bromate couple," Green Chemistry, vol. 10, no. 2, pp. 232-237, 2008.

[35] B. Sreedhar, P. S. Reddy, and M. Madhavi, "Rapid and catalystfree $\alpha$-halogenation of ketones using $N$-halosuccinamides in DMSO," Synthetic Communications, vol. 37, no. 23, pp. 41494156, 2007.

[36] C. Lucia, F. Maria, L. Paolo, and T. Francesco, "Ligandfree Suzuki coupling of arylboronic acids with methyl (e)-4bromobut-2-enoate: synthesis of unconventional cores of HIV-1 protease inhibitors," Organic Letters, vol. 14, no. 15, pp. 39283931, 2012.

[37] J. Qiu, L. Wang, M. Liu, Q. Shen, and J. Tang, "An efficient and simple protocol for a $\mathrm{PdCl}_{2}$-ligandless and additive-free Suzuki coupling reaction of aryl bromides," Tetrahedron Letters, vol. 52, no. 48, pp. 6489-6491, 2011.

[38] G.-P. Lu, K. R. Voigtritter, C. Cai, and B. H. Lipshutz, "Ligand effects on the stereochemical outcome of Suzuki-Miyaura couplings," The Journal of Organic Chemistry, vol. 77, no. 8, pp. 3700-3703, 2012.

[39] B. D. Gwenaelle, B. Agnes, K. A. John, B. Gerald, and Q. Anna, "Access and regioselective transformations of 6-substituted 4aryl-2, 8-dichloropyrido[3,2-d] pyrimidine compounds," The Journal of Organic Chemistry, vol. 77, no. 10, pp. 4586-4595, 2012.

[40] E. Vedejs and D. A. Barda, "Progress toward synthesis of diazonamide A. Preparation of a 3-(oxazol-5-yl)-4-trifluoromethylsulfonyloxyindole and its use in biaryl coupling reactions," Organic Letters, vol. 2, no. 8, pp. 1033-1035, 2000.

[41] G. A. Molander and T. Ito, "Cross-coupling reactions of potassium alkyltrifluoroborates with aryl and 1-alkenyl trifluoromethanesulfonates," Organic Letters, vol. 3, no. 3, pp. 393-396, 2001. 

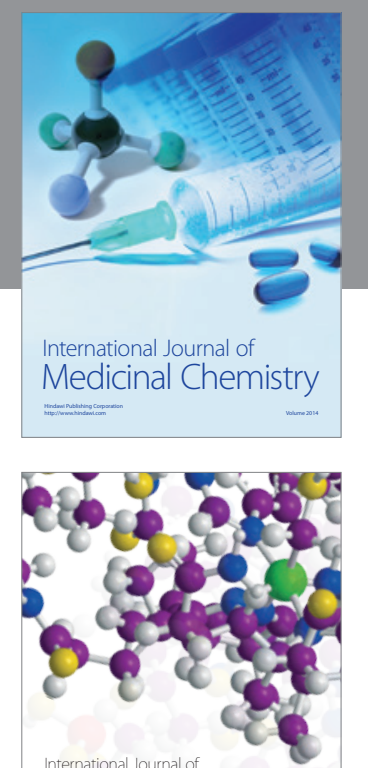

\section{Carbohydrate} Chemistry

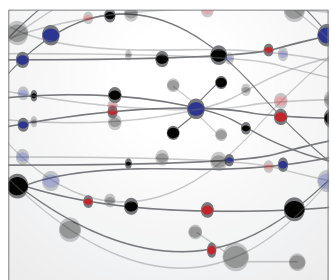

The Scientific World Journal
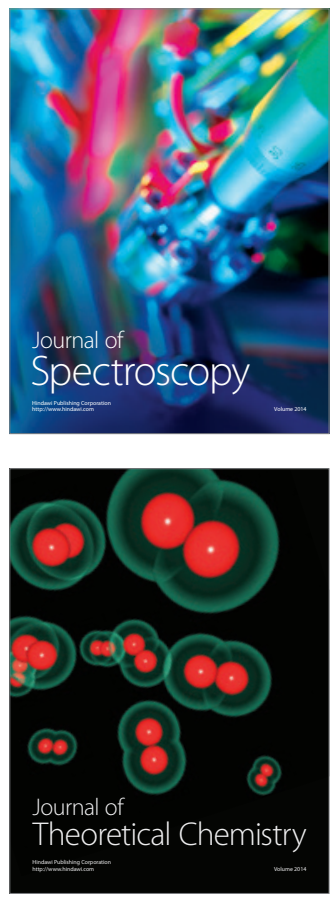
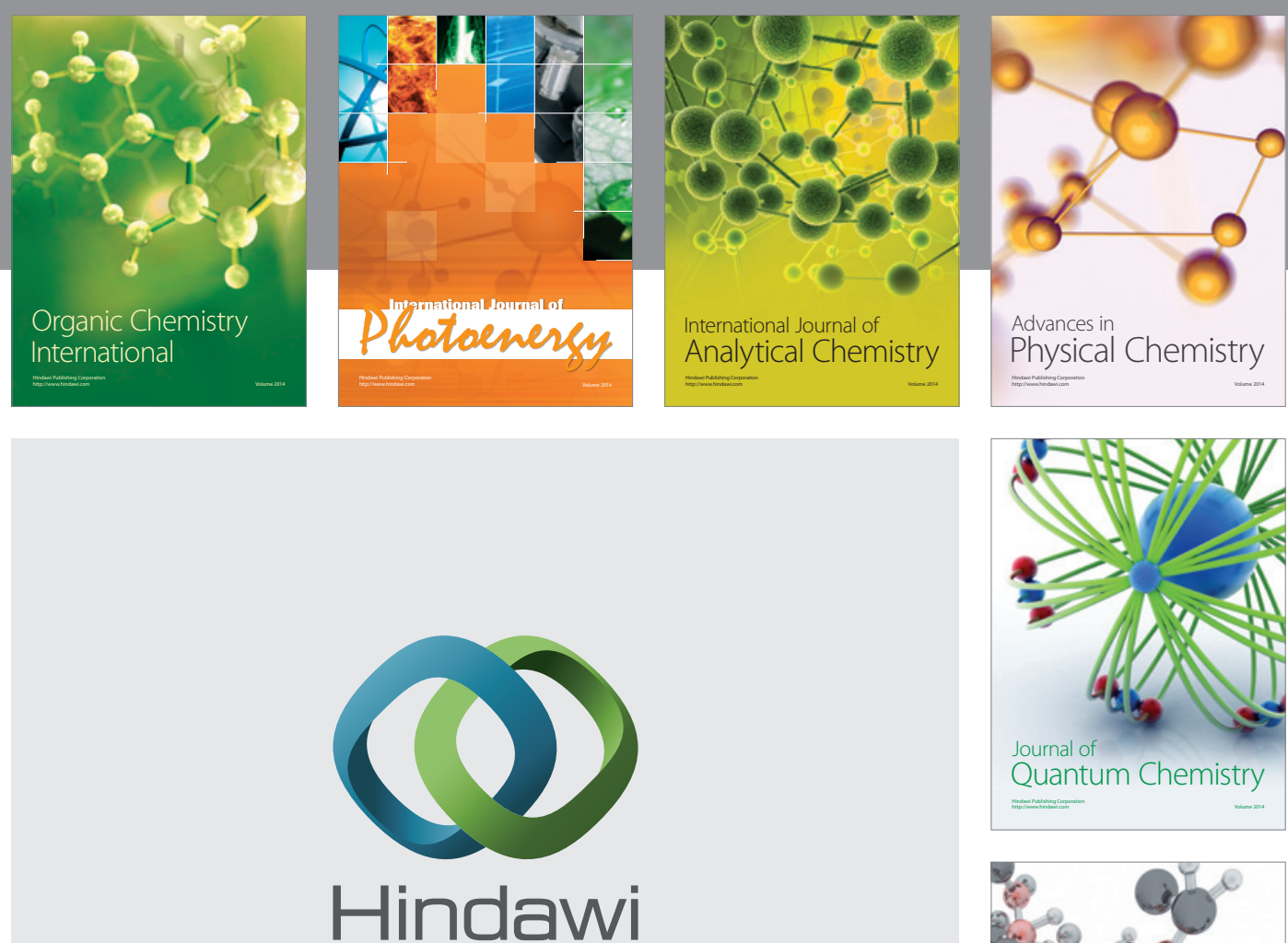

Submit your manuscripts at

http://www.hindawi.com

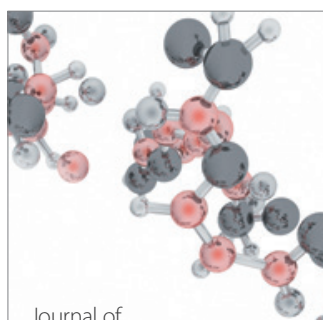

Analytical Methods

in Chemistry

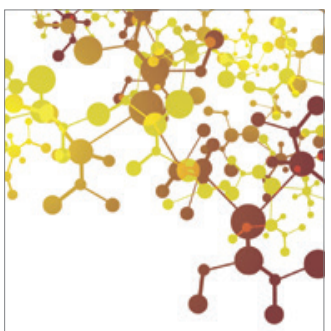

Journal of

Applied Chemistry

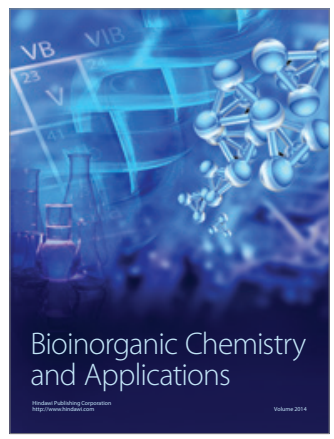

Inorganic Chemistry
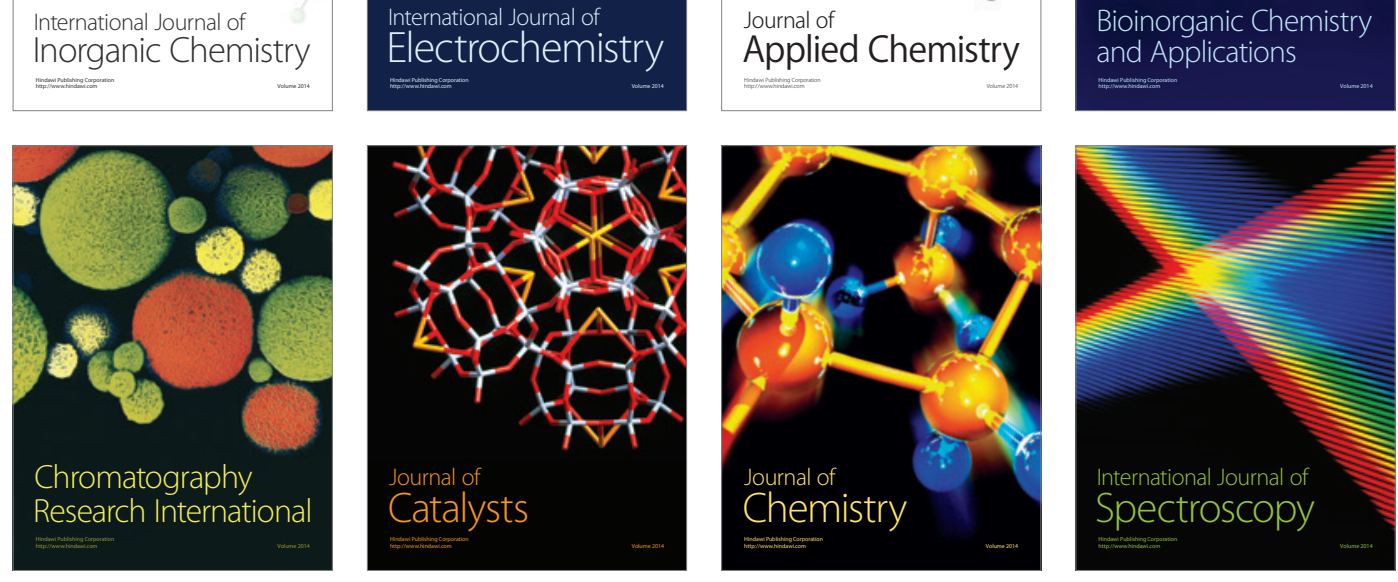